\title{
Géolinguistique
}

18 | 2018

Varia

\section{Du picard au français : commentaire d'un texte oral interlectal}

From Picard to French: Comment an Interlaced Oral Text

\section{Patrice Brasseur}

\section{(2) OpenEdition}

1 Journals

Édition électronique

URL : http://journals.openedition.org/geolinguistique/719

DOI : 10.4000/geolinguistique.719

ISSN : 2650-8176

Éditeur

UGA Éditions/Université Grenoble Alpes

\section{Édition imprimée}

ISBN : 978-2-37747-073-0

ISSN : 0761-9081

\section{Référence électronique}

Patrice Brasseur, «Du picard au français : commentaire d'un texte oral interlectal », Géolinguistique [En ligne], 18 | 2018, mis en ligne le 01 décembre 2018, consulté le 13 septembre 2019. URL : http:// journals.openedition.org/geolinguistique/719; DOI : 10.4000/geolinguistique.719

\section{Ce document a été généré automatiquement le 13 septembre 2019}

Géolinguistique 


\title{
Du picard au français : commentaire d'un texte oral interlectal
}

\author{
From Picard to French: Comment an Interlaced Oral Text
}

\author{
Patrice Brasseur
}

\section{Introduction}

\subsection{Le " rouchi », une variété de picard}

1 Le domaine linguistique picard couvre totalement ou en partie les départements du Nord, du Pas-de-Calais, de la Somme, de l'Oise et de l'Aisne et déborde assez largement en Belgique. J'ai choisi de m'intéresser pour cet article au parler de Gommegnies, qui se trouve à la périphérie orientale de l'aire d'extension du picard. Ce village a fait l'objet d'une enquête effectuée en 1964 par Claude Deparis, pour l'Atlas linguistique et ethnographique picard (désormais ALPic). Ce point d'enquête est cartographié dans cet ouvrage sous le numéro 54 .

$\mathrm{Du}$ fait de cette situation quelque peu marginale, les locuteurs de ce parler ne se perçoivent pas comme Picards, encore moins sous l'appellation de Chtis, abréviation d'un plus ancien Chtimi, qui semble née dans les années 1970 et a été largement diffusée depuis. Leur opinion s'appuie sur quelques faits morphologiques et phonétiques qui les différencient assez nettement du voisinage immédiat. C'est ainsi, par exemple, que la morphologie du parler de Gommegnies présente plusieurs différences notables avec l'ensemble picard en général. On n'y dit pas ch' cat pour « le chat », comme dans $75 \%$ des parlers picards, ni ch'l horloge comme dans le Pas-de-Calais, une grande partie de la Somme, mais l'cat l est dins l'horloge (expression traduisant le désaccord, spécialement au sein d'un couple, qui équivaut au français «le torchon brûle»). On n'y connaît pas non plus sin pour l'adjectif possessif «son », comme $80 \%$ des parlers picards, mais s', comme dans l'Avesnois et une partie du Valenciennois (voir les données de l'ALPic, cartes 9, 11, 13). Le parler de Gommegnies est, en effet, une forme de ce qu'Hécart a popularisé sous le nom de «rouchi » et qu'il fait venir d'une mauvaise coupure de les gens d'drouchi «les 
gens d'ici », prononcé d'ailleurs localement drauchi. (Pour une critique du terme rouchi, voir Deparis, 1978 : sans pagination.) Hécart définit ainsi les contours de cette variété de picard : «Le rouchi est parlé dans le ci-devant Hainaut français et une partie du Hainaut belge, jusqu'à Avesnes et Maubeuge. » (1978 [1834] : 415b)

Mais à l'intérieur de ce sous-ensemble du "rouchi », le village en question possède une originalité certaine. En effet, si ses voisins immédiats disent tous s' quien ou s' tchien « son chien", il semble le seul à dénasaliser $[\tilde{\varepsilon}]>[\mathrm{e}]: s^{\prime}$ tchiê $i$ queurt après mes glainnes "son chien court après mes poules ». Certes, les différences entre les divers parlers de l'Avesnois et du Valenciennois n'empêchent nullement l'intercompréhension. Cela n'empêche cependant pas les railleries vis-à-vis du village voisin d'Obies, par exemple, où l'on emploie nin " pas » comme deuxième terme de la négation, alors que les locuteurs locaux utilisent point ou mie, qu'ils ne manquent pas de juger plus élégants. Dire j'y vas nin «je n'y vais pas» signe la différence et quelques détails de ce genre permettent de distinguer aisément un natif d'un non-autochtone. On reconnaissait aussi facilement quelqu'un d'Englefontaine à sa façon de prononcer péun le mot " pomme », qui se dit pun à Gommegnies. Ces marques de différences, parfois subtiles, apparaissent d'ailleurs beaucoup mieux, dans la morphosyntaxe ou les réalisations phonétiques, que dans le lexique. Les anciens percevaient bien ce particularisme et l'avaient représenté dans une formule emblématique d'tévosé, bel et bé, mant caups, qui reprend trois locutions adverbiales dialectales et signifie « quelquefois, bel et bien, souvent ${ }^{1}{ }$.

\subsection{Le récit de $M$. Moine ; son caractère interlectal}

4 J'ai choisi d'étudier un enregistrement que j'ai moi-même recueilli dans ce village en 1977 auprès d'un locuteur natif, Henri Moine, sur le sujet de la fraude du tabac entre la Belgique et la France. Le texte est transcrit littéralement, dans une graphie qui respecte l'orthographe du français tout en gardant les traits spécifiques de la prononciation du locuteur. Chaque signe de ponctuation marque une rupture dans le débit. L'apostrophe signale une particularité phonétique. Les points de suspension notent spécialement une prolongation de la syllabe finale ou une hésitation sur la syllabe initiale. Les crochets entourent les interventions du transcripteur qui peuvent être des coupures (dues à des digressions ou des interruptions provoquées par des éléments externes), des explications nécessaires au lecteur privé de contexte, ou encore des notations de données non discursives telles que gestes ou rires. Les formes d'imparfait, qui seront analysées, ont été mises en gras. Les interventions de l'enquêteur (E.) sont en italiques.

5 M. Moine avait été scolarisé à l'école du village. Il connaissait donc le français, qu'il entendait quotidiennement à la radio et qu'il avait aussi l'occasion de lire dans les journaux. Mais, comme pour toutes les personnes de sa génération (il était né en 1897), cette connaissance restait probablement largement passive. Sortant peu, il ne conversait qu'avec ses voisins ou ses proches, notamment avec son épouse, présente au moment de l'enquête. La langue de ces interactions était le parler local. Il se livre ici à un exercice difficile inspiré du transcodage entre deux variétés qu'il connaît bien, mais dans des usages très différents. L'une est inhérente à sa vie quotidienne, l'autre est externe. Ce texte est donc une production située dans le temps et liée à la situation de communication.

6 Le récit dont il sera question n'est donc pas en français, ni en dialecte, même si les traits dialectaux ne manquent pas. La langue de ce locuteur ne doit pas être considérée comme 
un idiolecte insolite. Il s'agit d'une variété « interlectale » non normée, qui mêle les codes $\mathrm{du}$ français et du dialecte local, voire plus largement régional, et qui se manifeste de manière similaire chez tous les locuteurs. Les contours de cette variété restent flous, car si certains éléments de la langue sont toujours plus concernés que d'autres, la proportion de français et de dialecte est variable. En ce sens, chaque énoncé a donc ses caractéristiques propres. Ce fonctionnement est largement tributaire de la situation de communication : tout se passe comme si l'informateur faisait de son mieux pour parler le français, par respect pour l'interlocuteur qu'il ne connaît pas et qui, pense-t-il sans doute, risque de ne pas le comprendre autrement. Mais le contrôle reste approximatif et se relâche fréquemment dans le flux du discours.

7 Même si les observations que l'on peut faire sur ce texte ne sont sans doute pas extensibles à l'ensemble des locuteurs picards, les enquêtes que j'ai effectuées auprès d'une quinzaine de locuteurs à la fin des années 1970 dévoilaient des variétés interlectales similaires. Qui plus est, cette attitude qui était commune aux dialectophones de la génération de M. Moine, persiste encore chez les quelques locuteurs du parler local, comme j'ai pu le constater en octobre 2017.

8 L'intérêt du dialectologue se situe au-delà de cet exemple particulier et permet peut-être d'apporter une réponse à la question: quels traits dialectaux résistent le mieux au nivellement imposé par le français et passent au travers du filtre mis en place par le locuteur. C'est ce que je me propose d'étudier dans cet article.

\section{Transcription du texte oral étudié}

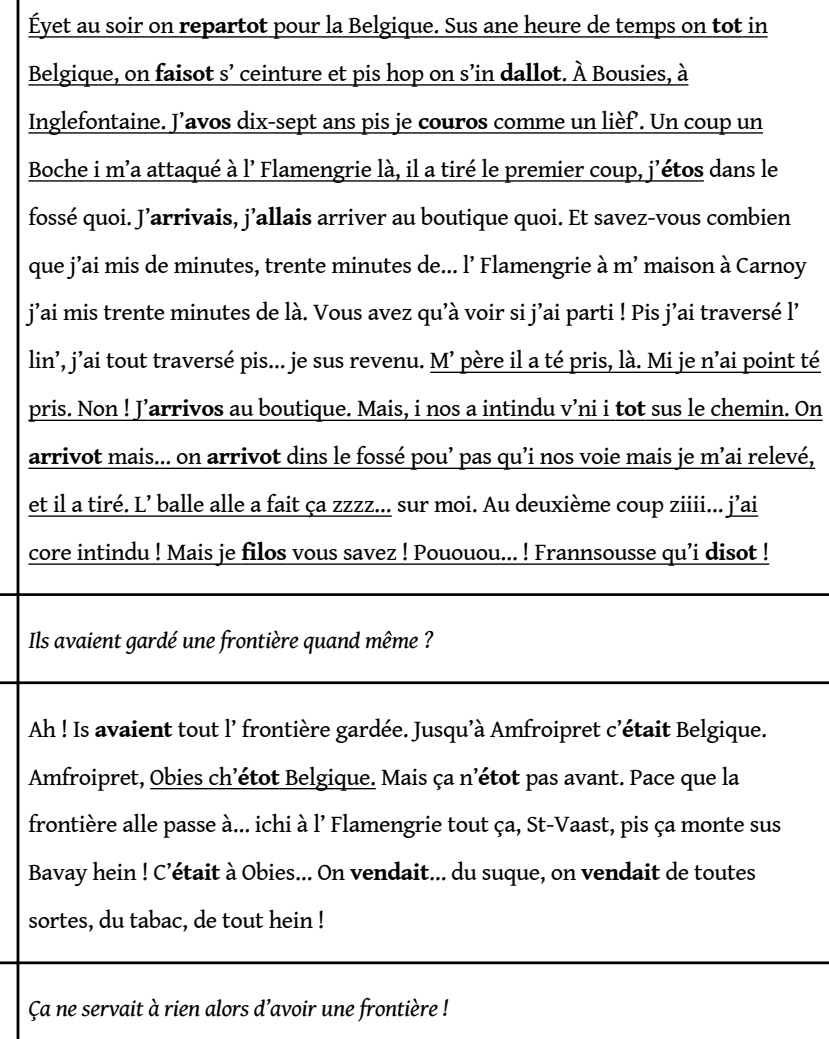




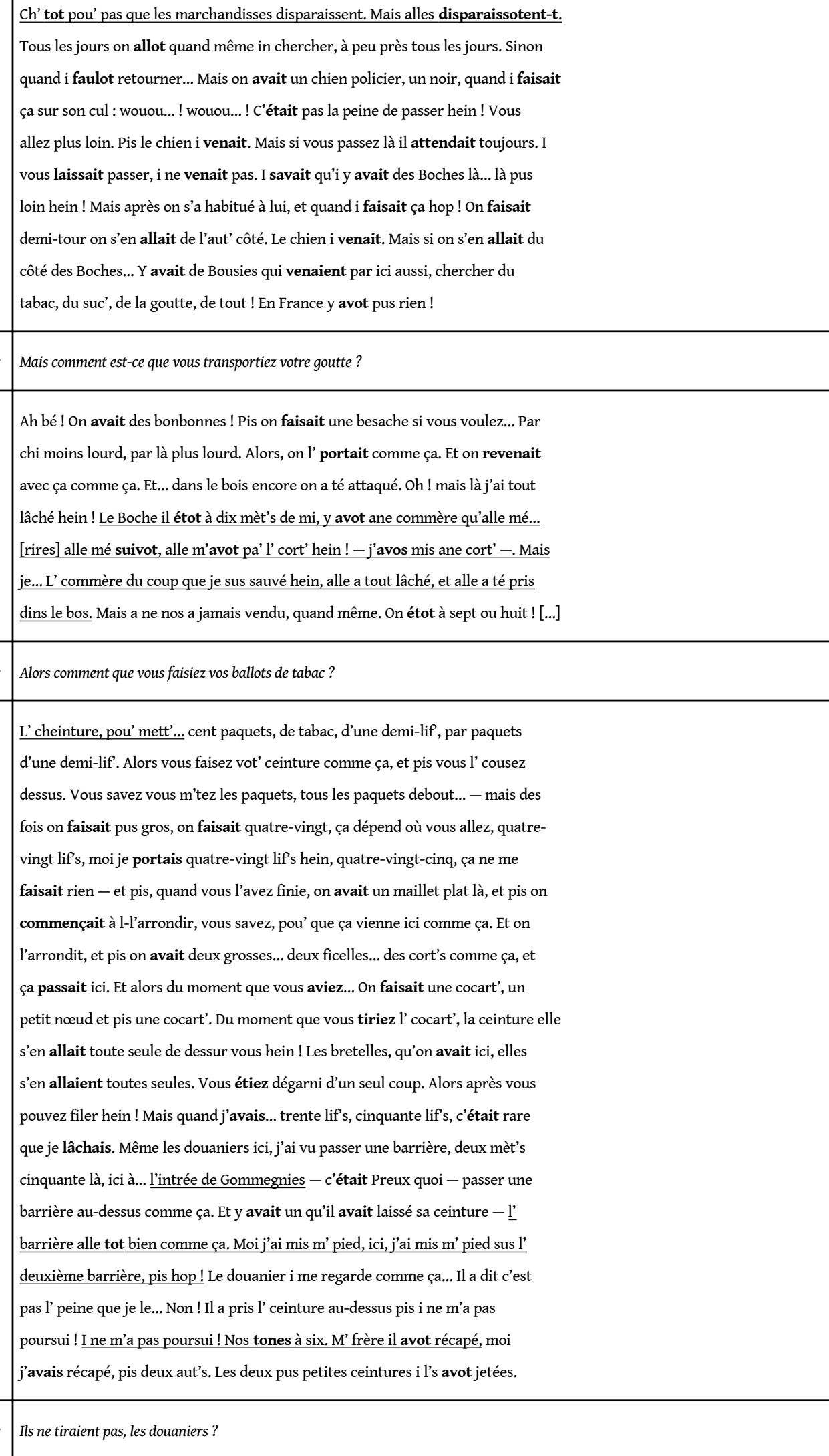


Ah! non ! Les douaniers jamais ! Les sommations is les f'saient ! À moi la bant' !

qu'is criotent ! À moi la bant' qu'is disuaient-t. Et pis is tiraient des coups de

revolver, mais en l'air hein ! Pou' appeler les aut's douaniers, si vous voulez. Mais

une demi-heure après où qu'on était?!

E. : Toujours la nuit hein! Quand il faisait clair de lune?

Ah! Non! Ane vraie lune on n'allait pas. Si ! Si on... avait passé une... une

ceinture ici supposons, on l' cachait le long de la rivière, on l' cachait... dans un

fossé. On mettait un peu d'herp' dessus, pis ça ne se voyait pas. Mais i fallait

toujours faire attention pour les traces que ça faisait. Pace que, le matin, si vous

voulez trouver une trace, c'est toujours le matin, vous verrez la trace tout de suite,

vous suivrez les contrebandiers à la trace. Mais on s'habituait à marcher sur un

chemin comme ça. Supposons, j'ai passé... On venait... de la Belgique des fois

- vous ne voudriez pas me croire ! - eh bien on passait un sentier là-bas à... l'

Boiscrette pis on suivait tout le chemin... depuis la Belgique jusqu'à ici, à

Gommegnies, on passait d'zous l'églisse de Gommegnies, vous m'entendez, on

arrivait là au Huyaume, y a un chemin qui revient sur l'égliss', ou... dans l' cache

de l'égliss'... On passait d'zous l'égliss', on passait sus l' place de Gommegnies,

on descendait l's escaliers, on suivait ici tout $t$-qu'en haut ici et on passait ici et

on s'en allait à Carnoy cacher ça. Sa ceinture. Qu'est-ce que vous me dites ! On

passait devant l' maison du brigadier ! C'est pas croyap' hein! Mais on avait le

passach'! On payait un douanier, on lui donnait s' dringuelle! [Rires]. Et y avait

6 un poteau, là, y avait un caillou, un gros grès, quand i n'y était pas fallait jamais

7 s'en aller. Une fois qué le caillou i tot retiré. Du moment qué... s'il était là sus le

78 côté fallait pas s'en aller !... On venait vers... huit heures du soir. Et pis on s'en

allait tout le long du chemin mais... Pou' s'en aller on n'avait rien! Les

douaniers is ne pouvaient pas... rien nous dire. Mais nos ceintures alles totent

tout le temps faites du jour d'avant. Et quand on avot le passach', hop! On

partait et allez ! Et on revenait tout le long du chemin comme ça pis y a une

femme un beau jour, elle vendait à boire, mais on n'allait jamais là à ce bistrot-là,

au coin, là - on l'appelait [X] [rires] elle nous a vendu mais le brigadier n'a pas

retrouvé nos ceintures. Quand on a su qu'i tot sus nos traces, poup! On a pris ses

86 ceintures, on l's a emportées dans le bois, et dans le bois i n'a jamais retrouvé nos ceintures hein! [...] Mais on ne mettait

87 jamais de tabac dans sa maison. Même

88 ma blaqu' je ne l'aurais pas mis, ma blaqu' de tabac! Oh! Non! On était

89 fraudeurs, mais fraudeurs raisonnap's hein! On n'était pas des bandits. C'était un

90 métier pour nous. On ne volait rien sur son passach' !

E. : Vous étiez beaucoup?

91 Sept, six, sept. On était des camarat's.

E.: Et y avait d'autres bandes? 
E. : Les marchands il n'y en avait pas ailleurs qu'à Bousies?

Ah! oui y en avait ! Y en avait plein les boutiques! Mais on avait toujours un

coin qu'on portait s' tabac, et personne n'en savait rien. Alors on prenait là son

tabac pis houp ! On savait qu'on pouvait partir, et pis on ne voyait jamais rien.

Mais quand on n'avait pas le passach'... Supposons on voulait passer

sus... Roisin là-bas, à la lin' de Roisin-Bavay, on allait là passer aussi. Eh bien !

On redescendait dans la lin', on suivait la lin' jusqu'à Saint-Vaast-la-Vallée. Et là

on remontait sur la plain-n' et pis on arrivait à la gare de Bavay, en face de la

gare. On arrivait là par... la plain-n', au long comme cha, y avait là une plain-n'.

Et nous passons à côté - là y avait des douaniers qui logeaient là dans la maison -

et nous passions dans la gare $-\mathrm{y}$ avait toujours des douaniers, mais ch' tot des

vieux douaniers - on passot dins la gare de Bavay tout $\mathrm{t}$-qu'au pont du chemin de

fer de l'aut' côté de la lin'... de la chaussée, et on traversot là dins les pâtures et

pis on s'en allait sur Mecquignies. Mecquignies on rintrot dans l' forêt et là on

cachot sus... le Coucou, on allait cacher là... à côté du Coucou et pis on

revenait. On avait des sentiers là qu'on passait dessus - on ne faisait pas de

traces hein ! Et toujours dans la nuit hein ! - et on se ramenait comme ça [...]

108 Ah non! Ch' tot des [X]... qu'on l's appelot. Ch' tot là qu'on dallot. Et pis à

109 Poix-du-Nord, qu'is étaient. Is achetaient tout dans tout. Ah! eux, tout de suite

110 payer ! Pour rien vous ne l'auriez pas eu hein! Ah! Non hein! Là on savait, on

111 était sûr qu'on pouvait y aller. Mais eux is l-l'emmenaient sur Saint-Quentin.

112 Eux is prenaient une voiture à chevaux de course, et pis houp ! L'zé v'là partis

113 avec les ceintures $[\ldots]$

E. : Sur la route ils ne pouvaient pas être pris!

114 Ah si ! Y avait core des douaniers, à Locquignol. Pace que vous avez... Au

115 Quesnoy y a des douaniers mais c'est des... mobiles si vous voulez, is vont

116 partout is vont à Obies, is vont n'importe où. Maintenant vous ne sauriez pus l'

117 faire, la fraut', mais is survellent maintenant je sais pas quoi... c'est le même prix,

118 ici que là-bas, alors... Ah ! À ce moment-là on gagnait sa vie quand on passait

119 une ceinture. Et ça tot pas co si cher que le tabac français. Et tout le monde amot

120 mieux le tabac bèch'. Mais y avot du bon tabac à ce temps-là ! Mais en derniers

121 temps cha n'allot pus. On nous f'sot du fourrach', avec du tabac. Bah! Cha ne tot

122 pus bon! Avec n'importe quoi on f'sait du tabac ! [...]

E. : Et du café, vous en preniez?

123 On d'avait quèquefois une lif' pour donner quèquefois à... un client qu'on vindot

124 s' tabac comme i faut vous savez. Quèquefois ane lif'. Quèquefois ane femme

125 qu'alle perdot pour cinquante... cinquante lif's de tabac. Mais i faulot payer tout

126 de suite. On avot des bons payeux.

E. : Que du tabac, vous ne preniez que du tabac? 
127 Du tabac! Mais pa' la guerre, pa' la guerre... de quarante, là on prenait du suc',

128 du tabac, des cigarettes, de toutes sortes, du... de l' castonat', tout ce qu'on

129 trouvait à revent'. On ne tot pas arrivé ici in France que... I n-y avot les [X] là

allez hop ! S'i faulot faire deux voyach's, is le faisotent-te par jour.

\section{Dialecte et français dans le texte}

\subsection{Lexique}

9 Je n'ai relevé dans le discours de M. Moine que quelques mots et emplois dialectaux : eyet (1) « et », s'in daller « s'en aller » (2), boutique (n. m.) « magasin d'alimentation » $(5,9,92)$, lin' (n. f.) « voie de chemin de fer» $(7,96,97,103)$, commère (n. f.) « jeune fille ; copine, bonne amie » $(31,33)$, bos (n. m.) « forêt » (34), récaper (v. intr.) « s'en tirer » (54), cache (n.f.) «sentier entre deux haies servant de raccourci»(70), dringuelle (n.f.) «pourboire» (75), vendre à boire « exercer le métier de cafetier» (83), pâture (n. f.) " pré » (103), daller « aller» $(2,108)$, savoir (116) « pouvoir », amer (119) « aimer », bèch' (120) «belge », castonat' (128) « cassonade », pa' $(127,127)$ « pendant », Harbénies (130) « Herbignies ».

10 Mais d'autres mots dialectaux attendus, couramment usités dans ce parler, manquent. Par exemple : toubaque « tabac » $(17,27,35,87,88,93,94,119,120,120,121,122,124,127,128)$, quère « chercher » $(26,130)$, lon (adv.) « loin » $(24)$, ruer « jeter », mucher « cacher », ê p'tit caup « un peu », fraudeux « contrebandiers ", vouée " sentier », toudis « toujours ", d'tévozé « quelquefois », Mecqu'nies « Mecquignies » (104).

\subsection{Phonétique : les voyelles}

11 - La terminaison - $a v u$ aboutit en français à $[u]$ et à Gommegnies, comme en picard ancien, à [0:]. Aucune attestation dialectale ne figure dans le texte, où caillou $(76,77)$ est prononcé en français.

12 - e ouvert suivi d'une entrave latine reste intact en français. Il se diphtongue à Gommegnies pour aboutir à [je]. Il n'y a aucune réalisation de ce trait sur 6 occurrences : sept $(3,34,91)$, bretelles (45), fer (102), cher (119).

$13-e$ fermé libre aboutit généralement à [wa] en français et à [o] à Gommegnies : là encore ce trait n'est pas représenté dans le texte à travers les 9 occurrences : soir (1), Carnoy [ Carpinetu] (6, 73), Amfroipret [< Auffridi pratu, localement Infropret] (13), noir (20), fois (37, 66), croire (67), soir (78), boire (83), Le Quesnoy [< Quercetu, prononcé localement Le Quéno ] (115).

N. B. : voir, qui se dit vir en picard, figure ici sous sa forme française (1. 7), tout comme quoi $(5,49,117,122)$, prononcé quoué dans le parler local.

14 - o fermé latin se combine avec un yod pour aboutir en français à [wa] et à Gommegnies à [o]. Une seule attestation apparaît dans le texte : bos $(34)$ [mais bois $(30,86)$ et Boiscrette (67)]. 
$15-e$ ouvert accentué entravé par nasale + consonne s'est combiné avec la nasale pour

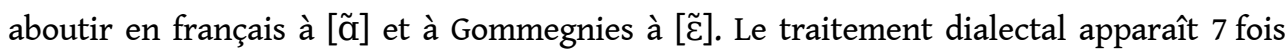
(hors en et dans): Inglefontaine [< Engeleri Fonte] (3), intindu « entendu» $(9,12)$, intrée (49), rintrer (104). Mais on relève 34 occurrences de la forme française: Amfroipret [prononcé localement Infopret] $(13,14)$, dépend (38), attendre, attention (63), bande [afr. bende] (3 fois), commencer, descendre, emmener, emporter, entendez (69), La Flamengrie [< Flamingeria] (3 fois), moment $(77,118)$, quarante [< lat. *quadraginta], redescendre, revendre, temps (4 fois), trente (47), vendre (4 fois).

$16-e$ ouvert accentué libre suivi d'une nasale finale ou devenue finale s'est combiné à elle

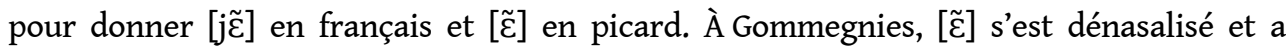
abouti à [e]. L'interjection ah bé! (28) n'a pas de caractère local. Par contre combien (5), rien $(27,39,79,80,90,93,94,110)$, chien $(19,21,24)$, bien $(51)$, eh bien $(67,96)$, revient $(70)$, qui seraient respectivement [kõbe], [Re:], [t]je:], [be], [ebe], [Rve:], restent en français.

17 - La combinaison de $e$ ouvert avec $l$ vocalisé aboutit à [o] en français, à [jo] en rouchi, mais à $[ø]$ à Gommegnies. Les deux mots concernés présentent la finale française : poteau (75), beau (83), même si, dans la prononciation du premier, on note une tendance certaine à la centralisation de la voyelle [o].

18 - Nasalisation régressive secondaire : plain.ne «plaine» $(98,98,99)$, sans doute ici par analogie avec le français plein/pleine.

\subsection{Phonétique : les consonnes}

19 - Le [r] final s'amuït dans plusieurs catégories de mots, illustrées ici par veni « venir » (9), co « (en)core » $(119)$, payeux « payeurs » $(126)$, mais faire $(63,117,131)$ et jour $(s)(19,19,81$, $82,131)$, toujours $(2263,64,92,101,107)$, fraudeurs $(89,89)$ restent sous leur forme française.

20 - En position finale [lj] se réduit ordinairement à [j] en français et à [1] à Gommegnies. Une seule attestation : survellent « (ils) surveillent » (117).

21 - En position finale devant [ə] (amuï), [n] est resté intact en français, mais a abouti à [n] à Gommegnies : un seul mot est concerné ; toutes les occurrences présentent la forme dialectale : lin' $(7,96,97,97,103)$.

22 - En position finale derrière une consonne, $[\mathrm{R}]$ et [1] tombent. Ce phénomène, qui n'est pas inconnu du français populaire, est systématique dans le parler local. Il peut se conjuguer ici avec le traitement relevé en 2.2.5: mèt' "mètre" $(31,48)$, mett' « mettre » (35), vot' " votre » (36), aut' « autre » $(25,55,58,103)$, revente « revendre » (129), suc' « sucre » $(16,27,127)$, lièfe «lièvre » (3), croyap" «croyable » (74), raisonnap' « raisonnable » (89).

23 - Les consonnes sonores [b], [d], [g], [z], [v], [3], en position finale devant [ə], ont abouti aux sourdes [p], [t], [k], [s], [f], [J] correspondantes. Ce phénomène, systématique dans le parler local, se réalise à $100 \%$ dans le texte : herp' « herbe » (62), croyap' " croyable » (74), raisonnap' " raisonnable » (89), cort' « corde » $(32,32,42)$, cocart' " cocarde » $(43,44,44)$, bant' "bande " $(56,57)$, camarat' " camarade » (91), fraut' "fraude » (117), castonate « cassonade » (128), revente « revendre » (129), blaqu' «blague » $(88,88)$, églisse « église » $(69,70,71,71)$, marchandisses « marchandises » (18), lièfe « lièvre » (3), lif " livre » $(35,36$, $39,39,47,47,123,124,125)$, passach' " passage » $(75,81,90,95)$, bèche «belge » (119), fourrach' « fourrage » (121), voyach' " voyage » (131). 
$24-c+a$ latin initial ou intérieur derrière consonne aboutit à [ [] en français, mais reste $[k]$ ici, comme dans toute la partie septentrionale du domaine normanno-picard: on relève deux attestations de ce traitement, récaper (54) et cache (70), cité dans la partie consacrée au lexique, dont l'équivalent français chasse n'a jamais l'emploi qu'il a dans le texte. Tous les autres mots concernés sont ici en français : chien $(19,21)$, chemin $(66,68,70,79,82$, 102), chaussée (103), acheter (109), cher (119).

$25-c+e$ ou $i$ latins initial ou intérieur derrière consonne et $c+y$ intérieur derrière voyelle aboutissent à [s] en français et à [S] à Gommegnies. Ce traitement, comme le précédent, est caractéristique des parlers picards; il est ici assez peu attesté (4 occurrences): cheinture (35) [contre 14 fois ceinture], besache «besace» (28), ichi (15), chi (29) [contre 14 fois ici]. On peut ajouter cache (70), pour le traitement de la consonne finale, avec la réserve exprimée ci-dessus. Mais suque $(16,26,127)$, cent (35), commencer (40), ficelle (42), cinquante $(47,49,125,125)$, trace $(63,64,85)$, place (71), chaussée (103), et cigarettes (128) se trouvent ici toujours sous la forme française.

Au total, donc, le système vocalique est celui du français, à la différence de ce qui se passe pour les consonnes. Pourquoi cette différence de traitement? Comme le dit Léon (2001: 92), «les consonnes sont surtout responsables de l'intelligibilité du discours et les voyelles de son audibilité ». Le locuteur donne ainsi à son discours le «son » du français, lui dénie tout particularisme local, en lui conservant cependant son caractère régional, largement identitaire. En effet, les traits consonantiques du parler local sont largement répandus dans l'ensemble dialectal picard. C'est le cas de la chute du $r$ final ainsi que du traitement de $c+a$ et de $c+e, i$ latins. Les autres traits - la démouillure d'un [lj] initial et de [n], la chute de $[\mathrm{R}]$ et [1] derrière consonne, le dévoisement des consonnes sonores finales - sont même adoptés par les locuteurs non dialectophones et peuvent dès lors être considérés comme du français régional. Rien de tout cela n'est spécifiquement local.

\subsection{Morphologie}

\subsubsection{Les articles}

\begin{tabular}{|l|l|}
\hline Formes françaises & Formes dialectales correspondantes \\
\hline un (22 attestations) & \\
\hline une (17 attest.) & ane $(1,31,32,60,124,124)$ \\
\hline la (28 attest.) & l'(23 attest.) \\
\hline \multicolumn{2}{|c|}{ le } \\
\hline \multicolumn{2}{|c|}{ les (devant consonne) } \\
\hline les (devant voyelle) (58) & l's (devant voyelle) (72) \\
\hline
\end{tabular}

Les formes précédées d'un astérisque ne sont pas attestées dans le texte. 


\subsubsection{L'adjectif possessif}

27 Ici encore les parlers dialectaux ne connaissent qu'une seule forme pour le masculin et le féminin, $m^{\prime}, t^{\prime}, s^{\prime}\left([\mathrm{m}],[\mathrm{t}]\right.$, [s]). Il n'y a aucune occurrence de la $2^{\mathrm{e}}$ personne dans le texte. Les formes dialectales et françaises se partagent à peu près également :

\begin{tabular}{|l|l|}
\hline Formes françaises & Formes dialectales correspondantes \\
\hline${ }^{*}$ mon & $m^{\prime}(8,51,51,54)$ \\
\hline$m a(88,88)$ & $m^{\prime}(6)$ \\
\hline son $(21,90,93)$ & $s^{\prime}(93,124)$ \\
\hline$s a(50,73,87,118)$ & $s^{\prime}(2,75)$ \\
\hline
\end{tabular}

\subsubsection{Les pronoms}

\begin{tabular}{|c|c|c|}
\hline & Formes françaises & Formes dialectales correspondantes \\
\hline $\begin{array}{l}1^{\mathrm{re}} \text { sg. sujet disjoint ou } \\
\text { complt prépositionnel }\end{array}$ & $\operatorname{moi}(11,39,51,54,56,57)$ & $m i(8,31)$ \\
\hline masculin $1^{\mathrm{er}}$ sg. COD & $m e(52,67)$ & mé $(31,32)$ \\
\hline masculin $1^{\mathrm{er}}$ sg. COI & $m e(39,73)$ & $*_{m e ́}$ \\
\hline f. $3^{e}$ sg. sujet (dev voy.) & & alle $(11,33,33)$ \\
\hline f. $3^{\mathrm{e}}$ sg. sujet (dev ${ }^{t}$ cons.) & & $a(34)$, alle $(15,31,32,32,51,125)$ \\
\hline f. $3^{\mathrm{e}}$ sg. COD & $* l a$ & $l^{\prime}(29,36,61,61,116)$ \\
\hline $3^{\mathrm{e}} \mathrm{sg}$. COI ou cplt préposit. & lui $(24,75)$ & $* l i$ \\
\hline $1^{\text {re }}$ pl. sujet & nous $(100,101)$ & $\operatorname{nos}(53)$ \\
\hline $1^{\text {re }} \mathrm{pl} . \mathrm{COD}$ & $*_{\text {nous }}$ & $\operatorname{nos}(9,10,34)$ \\
\hline $1^{\mathrm{re}} \mathrm{pl} . \mathrm{COI}$ & nous $(80,84,121)$ & \\
\hline $1^{\mathrm{re}}$ pl. complt préposit. & nous (90) & *nos \\
\hline $2^{\mathrm{e}}$ pl. sujet & vous (29 attest.) & *vos \\
\hline $2^{\mathrm{e}}$ pl. COD ou cplt préposit. & vous $(23,45)$ & *vos \\
\hline $\mathrm{m} .3^{\mathrm{e}}$ pl. sujet dev ${ }^{\mathrm{t}}$ voy. & $\operatorname{eux}(109,111,112)$ & $*(r)$ eusses \\
\hline f. $3^{\mathrm{e}}$ pl. sujet dev ${ }^{\mathrm{t}}$ voy. & elles (45) & alles $(18,80)$ \\
\hline
\end{tabular}




\begin{tabular}{|c|c|c|}
\hline $3^{\mathrm{e}} \mathrm{pl} . \mathrm{COD}$ & les (56) & l's $(55,88,108)$, l'zé (112) \\
\hline en & $\begin{array}{l}\text { en (14 attest., dont } 10 \text { pour } \\
\text { s'en aller) }\end{array}$ & $d^{\prime}(123)$ \\
\hline on & on (103 attest.) & $*_{i n}{ }^{*} \hat{e}$ \\
\hline$c e\left(\operatorname{dev}^{t}\right.$ v. être $)$ & $c^{\prime}(11$ attest.) & $c h^{\prime}(14,18,101,108,108)$ \\
\hline ce antécédt de que & tout ce que $(128,130)$ & *tout chu que \\
\hline$c ̧ a$ & $c ̧ a(26$ attest.) & cha $(99,121,121)$ \\
\hline
\end{tabular}

\subsubsection{Les prépositions}

Je ne note que les prépositions qui ont un équivalent dialectal dans le parler local :

\begin{tabular}{|l|l|}
\hline Formes françaises & Formes dialectales \\
\hline pour $(1,63,90,110,123,125)$ & pou' $(10,18,35,41,58,79)$ \\
\hline $\operatorname{sur}(11,21,65,70,90,98,104,111)$ & sus $(1,9,15,51,71,77,85,96,105)$ \\
\hline *sous & d'zous $(69,71)$ \\
\hline en $(27,58,72,120)$ & in $(129)$ \\
\hline dans $(13$ attest.) & dins $(9,34,102,103)$ \\
\hline jusque $(13,68,97)$ & tout t-que $(72,102)$ \\
\hline par $(26,28,29,35,99,131)^{2}$ & pa' $(32,127,127)$ \\
\hline devant $(74)$ & \\
\hline vers $(78)$ & \\
\hline du côté de (26) & \\
\hline
\end{tabular}

\subsubsection{Les formes verbales : I'imparfait}

\begin{tabular}{|l|l|l|}
\hline & \multicolumn{2}{|c|}{ Nombre d'attestations } \\
\hline & Formes françaises & Formes dialectales \\
\hline $1^{\text {re }}$ sg. & -ais (6) & -os (6) \\
\hline $2^{\text {e }}$ sg. & -ais (0) & -os (0) \\
\hline
\end{tabular}




\begin{tabular}{|l|l|l|}
\hline $3^{\mathrm{e}} \mathrm{sg}$. & - ait (117) & -ot (48) \\
\hline $1^{\mathrm{re}} \mathrm{pl}$. & - -ions (1) & -ones (1) \\
\hline $2^{\mathrm{e}} \mathrm{pl}$. & - iez (3) & -uaient- $t$, -otes $(0)$ \\
\hline $3^{\mathrm{e}} \mathrm{pl}$. & -aient (11) & -uaient-t (1), -otent- $t(5)$ \\
\hline
\end{tabular}

À l'imparfait (et au conditionnel) le parler de Gommegnies hésite entre des formes en $[ч \varepsilon]$ (ou $-[w \varepsilon])$ et en $-[o]^{3}$, soit pour le verbe canter « chanter » :

- [3 kãtuع] ou [3 kãto] ;

- [ty kãto];

- [i kãto] ;

- [no kãton] ;

- [vo kãttчtt] ou [vo kãtst] ;

• [i kã̃tчعt(te)] ou [i kãtot(te)].

Le conditionnel n'apparaît que 4 fois dans le texte et toujours sous sa forme standard.

Le locuteur a parfois quelques difficultés à manier la conjugaison française de l'imparfait, lorsqu'il doit employer des formes rares, comme celle de la $1^{\text {re }}$ personne du pluriel. Il substitue alors volontiers le présent. L'exemple le plus évident se trouve aux lignes 97-100 : «On arrivait là par... par la plaine, $\mathrm{y}$ avait là une plaine. Et nous passons à côté - là y avait des douaniers qui logeaient là dans la maison - et nous passions dans la gare $-\mathrm{y}$ avait toujours des douaniers, mais che tot des vieux douaniers - on passot dins la gare de Bavay [...].» Dans un récit à l'imparfait, la forme de présent nous passons, sans doute attirée par le dialectal nos passones, témoigne de l'embarras du locuteur, qui reprend très vite par la forme correcte nous passions, mais élude le problème avec l'emploi de on passot.

Un examen du texte d'un point de vue pragmatique ne permet pas d'expliquer de manière satisfaisante le maintien de certaines formes dialectales puisque, outre des énoncés où les deux éléments sont dialectaux comme ch'étot (14), che tot $(17,101,108)$, cha ne tot pus (121), alle tot (50), alles totent (80), ou français comme on avait $(28)$, y en avait $(92,92)$, c'était $(20$, $47,49,89)$, on était $(59,88,110)$, on n'était pas $(89)$, on relève tout autant d'énoncés hybrides : on étot (34), on ne tot pas (129), ça tot (119), ça n'étot pas (14) ou encore on d'avait (123). Ceci est sans doute dû à la forte prégnance du système dialectal dans la conscience linguistique du locuteur.

La morphologie flexionnelle de l'indicatif imparfait est particulièrement complexe dans les parlers picards de l'est. Les rares données comparables sur le sujet (ALPic 639 « ils avaient », 640 " (nous) étions »; $A L W^{4}$ 2-110 «(nous) savions », 2-112 «(ils) devaient ») montrent que les formes d'imparfait sont très nombreuses en picard moderne. Pour situer le parler de Gommegnies dans cet ensemble, nous avons repris les données des atlas et placé le village au centre d'une aire d'un rayon d'une quarantaine de kilomètres. 
ALPic 639 « ils avaient » et ALW 2-112 « ils devaient ».

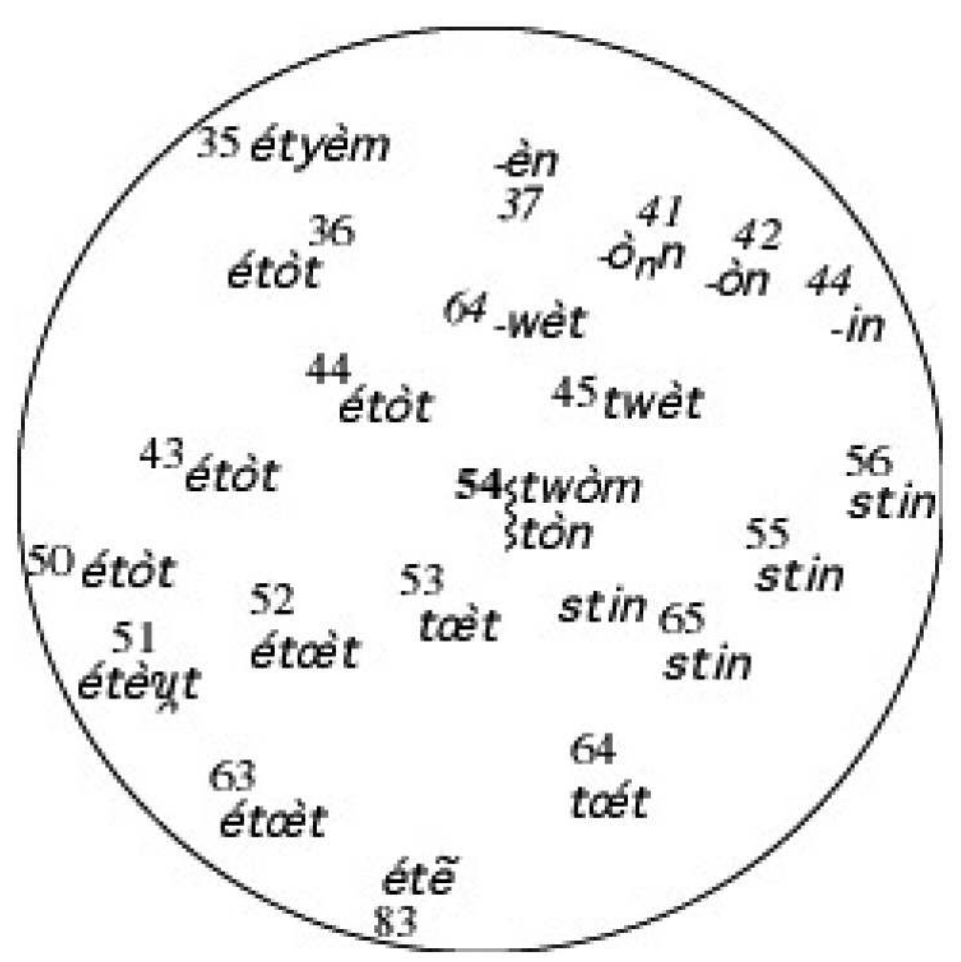

ALPic $640^{5}$ « nous étions » et $A L W$ 2-110 « nous savions ».

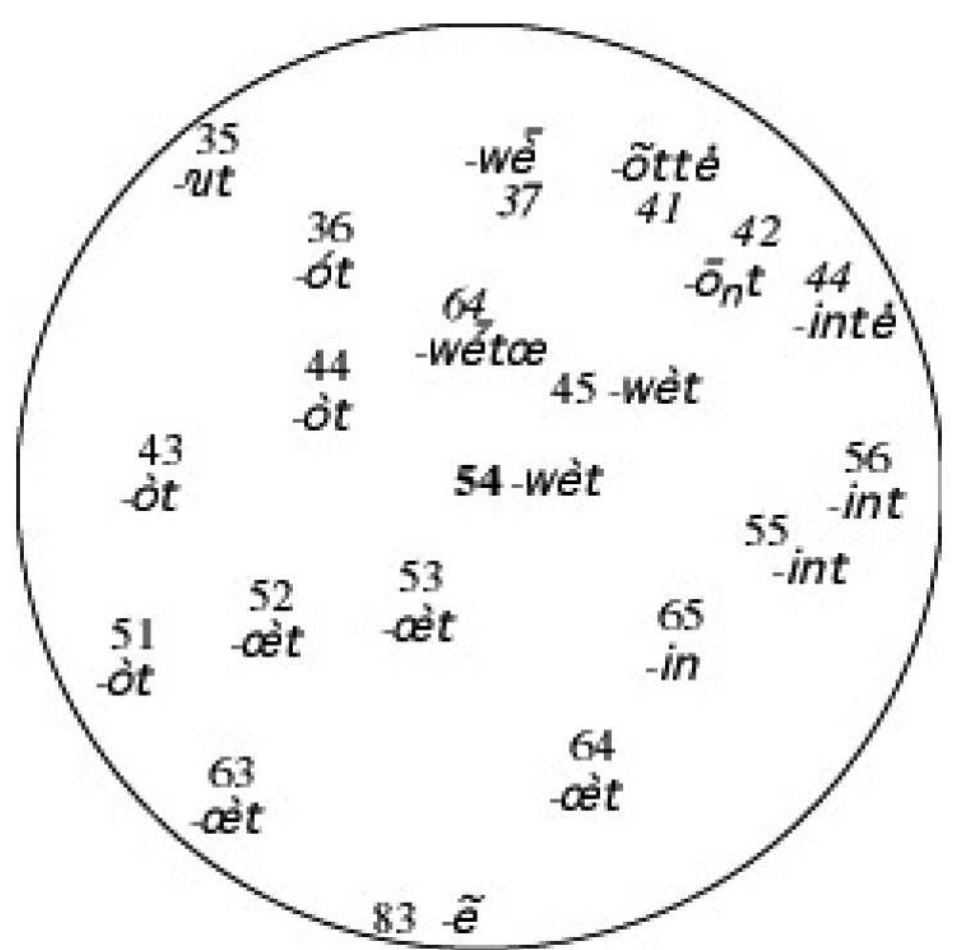

34 Pour ce qui concerne la $1^{\text {re }}$ personne du pluriel, le parler local, avec ses formes en -[on] ou -[wom], se situe au point de rencontre entre la limite de l'aire picarde de -[ot] (et de sa variante -[œt]) et de l'aire wallonne de -[in] (et de sa variante -[عt]. Ces formes en [on] sont seulement attestées dans deux autres points belges appartenant au parler 
rouchi et forment, en quelque sorte, une zone-tampon entre les deux vastes aires précitées. Les faits se rapportant à la $2^{\mathrm{e}}$ personne du pluriel sont parallèles, avec d'un côté des formes picardes en -[jt] et de l'autres des formes wallonnes en -[int].

On comprend mieux pourquoi le locuteur n'emploie ici à la $1^{\mathrm{re}}$ personne du singulier dialectal que des formes en -[o] et ne laisse échapper à la $3^{e}$ du pluriel que disuaient'te (57), contre disparaissotent (18), criotent (57), totent « étaient 》 (80), venotent'te (130), faisotent'te (131). Ceci est tout à fait conforme à son désir de parler français dans l'interaction : il commence en quelque sorte par éliminer les formes spécifiques avant de s'attaquer aux formes beaucoup plus courantes régionalement et qui le singularisent moins.

\subsubsection{Autres formes verbales notables}

- té « été » (du v. être) $(8,8,30,33)$;

- je sus (du v. être) $(8,33)$;

- je m'ai relevé (10), on s'a habitué (24),j'ai passé (66) ;

- poursui « poursuivi » (53);

- faulot «fallait » $(20,125,131)$;

- perdot « prenait » (125) [mais prenait aux lignes 93 et 127];

- vous faisez « vous faites » (36);

- vous m'tez « vous mettez » (37);

- i-n'y avot « il y avait » (29).

Il faut cependant noter que toutes les autres formes verbales, notamment les subjonctifs (en -[S] en picard) ont la forme du français: pou pas qu'i nos voie (10), pou pas que les marchandisses disparaissent (17), pou' que ça vienne (41).

- aurais (88).

\subsubsection{Règles d'accord}

Je relève deux attestations du non-accord au féminin, qui sont tout à fait conformes à l'usage du parler local : is avaient tout l' frontière gardée (13) et alle a té pris (33).

\section{Conclusion}

On a bien vu que des formes appartenant à deux variétés linguistiques coexistent dans ce texte : celles du français commun, à maints égards la langue cible du locuteur et celles du dialecte picard (ici sous sa forme « rouchi »).

J'aurais aussi pu prendre en compte dans ces développements le caractère populaire de la langue du texte. Cet aspect est évidemment présent dans le dialecte, mais aussi dans le français du locuteur :

- prothèse vocaliques diverses, notamment : [əl], [el] «le », [əd], [ed] « de »;

- reprise systématique du pronom personnel après le sujet : « un Boche i m'a attaqué » (3), «me père il a té pris » (8), «le balle alle a fait ça » (10), « la frontière alle passe »(15), «le chien i venait » (21), etc. ;

- confusion de qui et qu'il : «y avait un qu'il avait laissé sa ceinture » (50);

- utilisation de formes comme un coup (3) « une fois », (et) pis (2, 37, 15, 21, 36, 39, 40, etc.), core « encore » $(11,114)$, vous faisez (36); 
- généralisation de l'auxiliaire avoir aux dépens de être, au passé composé, avec les verbes de mouvement ou d'état et avec les verbes pronominaux : j'ai parti (7), je m'ai relevé (10).

Je ne m'attarderai pas sur les causes de cet incessant va-et-vient entre ces variétés. Il s'agit véritablement ici d'un mélange de codes qui obéit à des ressorts complexes, largement dépendants sans doute de la situation de communication : l'enquêteur est un jeune enseignant ; l'enquêté est un vieil ouvrier; les deux viennent de se rencontrer, ne se connaissent que par personnes interposées.

Reprenons les grandes divisions de cet exposé pour examiner quels points du système dialectal résistent le mieux.

Nous l'avons vu, le lexique est peu spécifique. Les mots dialectaux employés sont, cependant, plus nombreux que ceux qui passent au français, sans doute pour deux raisons : certains (boutique, ligne, bois, pâture, vendre à boire) passent facilement inaperçus, car ils ne diffèrent du français que par leur emploi, d'autres (commère, cache, dringuelle) n'ont pas véritablement d'équivalent français, d'autres encore (eyet, daller, amer, castonate) ne diffèrent que par une légère différence phonétique sans régularité observable. À l'inverse, les mots dialectaux évités ont un équivalent français facilement identifiable ( quère, lon, ruer, fraudeux), sont même fortement emblématiques (mucher, toudis, d'tévozé) ou diffèrent nettement d'un point de vue phonétique (toubaque, ê p'tit caup, vouée).

2 Les traits phonétiques dialectaux sont diversement représentés dans le texte. Pour ce qui est des voyelles, un seul, largement répandu dans les parlers de la région, est bien

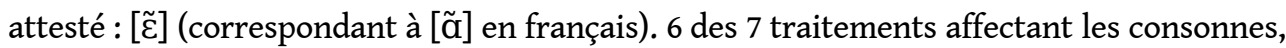
bien connus dans une vaste aire picarde, sont très bien représentés. Seul le maintien du $c$ dur latin, le trait le plus typique, est faiblement attesté. Nous pouvons sans doute donner à cette constatation une explication simple : les voyelles, si ce n'est $u$ et $i$ sont les sons les plus audibles et les plus intelligibles, comme le rappelle Straka (1979 : 62-63). Ici encore, donc, les traits les plus saillants sont éliminés.

La morphologie est sans doute la partie la plus facile à étudier, car les observations sont nombreuses. On a pu voir que la neutralisation de l'article défini (et de l'adjectif possessif), ainsi que de son corollaire, le pronom complément d'objet direct (français, le, la) notamment, semble le trait dialectal le plus résistant. Parmi les autres pronoms dialectaux, on relève aussi un large emploi de alle(s) " elle(s) » ainsi que in " en » et che "ce", tandis que on (régionalement in, localement $\hat{e}$ ) reste toujours français. Les prépositions figurent aussi très largement sous une forme dialectale, largement régionale. Remarquons enfin que le locuteur choisit les formes verbales de l'imparfait les moins caractéristiques du parler local.

44 Il est donc tout à fait clair que le locuteur, à la recherche d'une sorte de consensus linguistique, est conduit à éliminer les traits les plus saillants. Les faits dialectaux spécifiques ne passent pas la barrière du filtre, mais la morphologie, qui est au cœur du système linguistique, semble assez largement y échapper. 


\section{BIBLIOGRAPHIE}

BRASSEUR Patrice, 1970, « Le parler des sabotiers de Gommegnies (Nord) », Linguistique picarde, $\mathrm{n}^{\circ}$ 35, p. 2-7.

BRASSEUR Patrice, 1972, «Les noms des oiseaux sauvages dans le parler de Gommegnies (Nord) », Linguistique picarde, $\mathrm{n}^{\circ} 43$, p. 11-13.

BRASSEUR Patrice, 1981, « À propos de trois textes électoraux picards », dans D. Kremer et H.-J. Niederehe (éds), Littératures et langues dialectales françaises, Actes du colloque de Trèves (17-19 mai 1979), Romanistik in geschichte und gegenwart, $\mathrm{n}^{\circ} 10, \mathrm{p} .35-47$.

CARTON Fernand \& LEBÈGUE Maurice, 1989/1997 Atlas linguistique et ethnographique picard, Paris, Éd. du CNRS, t. 1 (cartes 1-317) / t. 2 (cartes 318-660).

DAUBY Jean, 1979, Le livre du « rouchi », parler picard de Valenciennes, Amiens, Musée de Picardie.

DAUBY Jean, 1983, Complément au livre du « rouchi », parler picard de Valenciennes, Amiens, Musée de Picardie.

DEPARIS Claude, 1978, « Préface » à la réimpression du Dictionnaire rouchi-français de G.-A. Hécart [1834], Marseille, Laffitte reprint.

HÉCART Gabriel-Antoine, 1978, Dictionnaire rouchi-français [1834, $3^{\mathrm{e}}$ éd.], Marseille, Laffitte reprints.

FLUTRE Louis-Ferdinand, 1977, Du moyen picard au picard moderne, Amiens, Musée de Picardie.

LÉON Pierre R., 2001, Phonétisme et prononciations du français, Paris, Nathan/VUEF.

REMACLE Louis, 1969, Atlas linguistique de la Wallonie (4 éd.), t. 2 : Aspects morphologiques, Liège, Vaillant-Carmann.

STRAKA Georges, 1979, Les sons et les mots, Paris, Klincksieck.

\section{NOTES}

1. Les lettres de noblesse de d'tévosé sont anciennes, et Froissart écrit de telles fois est «quelquefois».

2. Les emplois dialectaux de par et $p a$ ' sont distincts. Les locuteurs dialectophones disent en effet par chi « par ici » et par là (28-29), « par paquets » (35), ou par jour (131), mais « pa' la plaine » et non pas «par la plaine» (99), comme ici. Ces emplois sont décrits par Hécart (1834 : 331-332, s. v. pa) et Dauby (1979: 178, s. v. pa et 180, s. v. par).

3. Les formes en -[uع] apparaissent comme archaïques, plus spécifiques du parler local.

4. Les points de l'ALW, situés dans la province de Hainaut, arrondissement de Mons, sont en italiques. Gommegnies, point 54 de l'ALPic, est en gras. Nous ne donnons ici que les morphèmes dérivationnels en conservant l'alphabet phonétique des atlas linguistiques.

5. L'ALPic donne la forme twòm pour «(nous) étions", alors que nos propres enquêtes font apparaître plus fréquemment tòn. 


\section{RÉSUMÉS}

Cet article se propose d'étudier le récit d'un contrebandier, enregistré à Gommegnies (Nord) en 1977. Le parler de ce village, situé à la frontière belge, dans le domaine linguistique picard, a fait l'objet d'une enquête pour l'Atlas linguistique et ethnographique picard, où il figure sous le numéro 54. Le locuteur, qui s'efforce de parler le français, laisse cependant échapper de nombreuses formes dialectales, qu'elles concernent le lexique, la phonétique ou la morphologie. Quels éléments passent au travers du filtre mis en place par le locuteur? Quelles en sont les caractéristiques?

This paper aims to study the story of a smuggler, registered in Gommegnies (North) in 1977. The dialect of this village, located on the Belgian border, in the Picard language area, was the subject of an investigation for the Picard linguistic atlas, where it appears under number 54 . The speaker strives to speak French, but misses many dialectal forms, whether they concern lexicon, phonetics or morphology. Which elements pass through the filter set up by the speaker? What are their characteristics?

\section{INDEX}

Mots-clés : picard, dialecte, atlas

Keywords : Picard, dialect, atlas

\section{AUTEUR}

\section{PATRICE BRASSEUR}

Université d'Avignon 